\title{
A Theoretical Approach to Estimating Bird Risk of Collision with Wind Turbines Where Empirical Flight Activity Data Are Lacking
}

\author{
Robert W. Furness ${ }^{1}$, Mark Trinder', David MacArthur ${ }^{1}$, Andrew Douse ${ }^{2}$ \\ ${ }^{1}$ MacArthur Green, Glasgow, UK \\ ${ }^{2}$ Scottish Natural Heritage, Great Glen House, Inverness, UK \\ Email: bob.furness@macarthurgreen.com
}

Received 8 January 2016; accepted 5 April 2016; published 8 April 2016

Copyright (C) 2016 by authors and Scientific Research Publishing Inc.

This work is licensed under the Creative Commons Attribution International License (CC BY). http://creativecommons.org/licenses/by/4.0/

(c) (i) Open Access

\begin{abstract}
There are standard procedures for collecting data on numbers of birds at sites being proposed for wind farm development and evaluating collision risk for each key species. However, methods do not work well for all species. Where a local bird population is depleted, empirical data cannot provide estimates of likely collision mortality numbers if that population returns to satisfactory conservation status. Field survey methods are also inadequate for cryptic bird species. Both these problems can be important for evaluation of impacts of proposed wind farms on bird populations protected by the EU Birds Directive. We present an alternative method, based on energy constrained activity budgets and natural history, which permits assessment of likely collision numbers where empirical data are inadequate. Two case studies are presented where this approach has been successfully used to resolve disputed planning applications, one for a hen harrier population where numbers present are much below the population size at designation, and one for a cryptic species (greenshank). Our novel method helps reduce uncertainty in assessments constrained by difficulties in collecting suitable empirical data.
\end{abstract}

\section{Keywords}

Wind Farm, Collision Risk Modelling, Activity Budget, Flight, Birds Directive

\section{Introduction}

The European Union (EU) requires at least 20\% of its total energy needs to be produced by renewable sources

How to cite this paper: Furness, R.W., Trinder, M., MacArthur, D. and Douse, A. (2016) A Theoretical Approach to Estimating Bird Risk of Collision with Wind Turbines Where Empirical Flight Activity Data Are Lacking. Energy and Power Engineering, 8, 183-194. http://dx.doi.org/10.4236/epe.2016.84017 
by 2020 [1], and the UK government is legally committed to meeting $15 \%$ of the UK's energy needs from renewables by 2020 [1]. Member states may aim higher than the threshold set by the EU. Germany aims to produce $45 \%$ of its energy needs from renewable sources by 2030 [2]. Wind farms provide a major part of the strategy to increase energy production from renewable sources. Germany installed $5.2 \mathrm{GW}$ of wind power capacity in 2014, and the UK installed $1.7 \mathrm{GW}$, contributing to an increase of $11.8 \mathrm{GW}$ across the EU [3].

Although making a major contribution to reducing emissions of carbon dioxide, wind farms represent a hazard for some bird populations of conservation importance, through risk of collision with turbine blades, barrier effects, and habitat loss through displacement [4] [5]. Some proposed wind farms may have predicted impacts on bird populations protected under EU law by Special Protection Areas (SPAs) designated by Member States under the Birds Directive (2009/147/EC). Where a proposed wind farm may impact upon an SPA population, a Habitats Regulations Assessment (HRA) is required under planning law. Similarly, many bird populations in the wider countryside (with no association with SPAs) receive greater protection due to their status as Annex 1 birds under the Birds Directive. Where a proposed wind farm may impact on an Annex 1 species in the wider countryside, an Ecological Impact Assessment (EcIA) is often required; an obligation which originates from the Environmental Impact Assessment Directive (2011/92/EU).

Guidance from UK Statutory Nature Conservation Bodies (SNCBs) is for studies of bird flight activity at proposed wind farms to be carried out by Vantage Point (VP) surveys of bird activity in order to assess numbers of birds at risk of collision mortality [6]. Where sensitive bird species exist, such surveys should be carried out throughout the year, over a period of at least one and often two years, with observations made for a minimum of 36 hours per season (e.g. breeding, non-breeding, migratory) per VP, and using multiple VPs to provide adequate visual coverage of the entire proposed wind farm area [6].

HRA and EcIA can be complicated, and potentially compromised, for several reasons. HRAs can be particularly affected by the SPA bird population being in unfavourable condition (e.g. the population size being lower than the reference level for which the SPA was designated). In such circumstances, it is necessary to establish if the development may affect the population's ability to recover. If the birds are absent, then VP watches cannot establish risks of collision mortality, compromising such assessment. A good example of this problem is provided by hen harrier Circus cyaneus, a species on Annex 1 of the Birds Directive that breeds in upland habitat where many wind farms have been (and are proposed to be) situated in the UK. Fielding et al. [7] identified that conservation status of hen harrier was "Unfavourable" throughout England and Wales, and in 15 out of 20 Natural Heritage Zones in Scotland. The hen harrier population of Bowland Fells SPA was "at least 12 pairs" in the years 1986-90 immediately before SPA designation [8], but no hen harriers bred there in 2012 [9]. Similarly, the species has been lost from North Pennine Moors SPA [9], and the conservation status of hen harrier is now classified as Unfavourable at Forest of Clunie SPA, Langholm-Newcastleton Hills SPA, and Muirkirk and North Lowther Uplands SPA due to large declines in breeding numbers [10].

Proposed wind farms close to these SPA populations (defined as $<2 \mathrm{~km}$ by Scottish Natural Heritage, SNH) require HRA, but VP watches are unlikely to record levels of hen harrier flight activity representative of the designated populations because numbers are severely depleted. SNH initially objected to a wind farm proposal at Stranoch because there was insufficient information to properly assess the impact of collision mortality on the hen harrier populations of Glen App and Galloway Moors SPA. That SPA was designated on the basis of 10 breeding female hen harriers on average between 1994 and 1998, but survey work for the wind farm developers was carried out in 2009-2011 when only a single female hen harrier bred on the SPA. Survey data suggested that only 0.015 hen harriers would collide with turbines per year and therefore that the wind farm would have no impact on the integrity of the SPA. SNH rejected that argument on the grounds that the impact when the hen harrier population was at the designated population size was the relevant impact to address and not the impact when the population was close to local extinction.

That led the consultants for that project, MacArthur Green, to develop the modelling work described in this paper. The same approach was also used successfully for another wind farm (Kennoxhead) near the Muirkirk and North Lowther Uplands SPA where hen harrier breeding numbers had fallen from 29.2 females at designation to 11.7 in 2009-11 when VP surveys were carried out.

Some bird species are highly cryptic and therefore liable to be under-recorded in VP surveys of flight activity. For example, many flights by merlins Falco columbarius and by breeding waders are not detected during VP watches [6]. A recent wind farm proposal at Strathy South, Scotland, adjacent to an SPA with greenshank Tringanebularia as a feature, was supported by VP watches that recorded very few greenshank flights despite large 
numbers of greenshanks known to be nesting in the area. This anomaly led SNH to question whether collision risk may have been underestimated. SNH used the approach developed for hen harrier to provide a theoretical estimate of the collision risk for greenshanks at Strathy South in order to inform their position on that planning application which had gone to public enquiry.

Case law (Case C-127/02 "the Waddenzee Case") has concluded that all aspects of plans or projects which can affect an SPA's conservation objectives must be identified, using the "best scientific knowledge in the field" and that "...the competent national authorities ... are to authorise such activity only if they have made certain that it will not adversely affect the integrity of that site. That is the case where no reasonable scientific doubt remains as to the absence of such effects”.

Reaching a conclusion that a development will not affect site integrity may be impossible where that decision has to be based on compromised VP data. In this paper we outline a theoretical approach to estimating the amount of flight activity likely to occur at a proposed wind farm. We do not suggest that this novel approach should replace VP studies that provide empirical data, but rather that our theoretical approach may help to provide an alternative where empirical data appear to be compromised either by problems of low detectability, or by depleted numbers of birds in the study population. Our theoretical approach is founded on the metabolic constraint to flight activity in birds which sets a ceiling on flight activity levels, coupled with information on seasonality of activity budgets, and scenario modelling of population densities and home range use.

This approach led to SNH withdrawing its objections to the Stranochand Kennoxhead wind farms by providing confidence that it would not affect the integrity of the respective SPAs and was presented by SNH to the public enquiry for Strathy South as a key part of their case regarding the adverse impact of that proposed development on the greenshank population of Caithness and Sutherland Peatlands SPA. It has therefore played a key role in decision making for three recent planning applications for wind farms in Scotland where empirical data were judged inadequate.

Scenario modelling may have a number of useful applications for developers and SNCBs: it could be used as an early screening tool in the assessment of sensitivity of a site to collisions affecting bird populations, it could be used to increase the robustness of EcIAs and HRAs, and an agreed standardised approach (such as this) could help to reduce uncertainty in impact assessments, and therefore help to reduce delays in planning.

\section{Modelling Approach}

\subsection{The Concept of a Metabolic Ceiling to Time Spent Flying}

A constraint on flight activity that can be used to set an upper limit on activity levels is the energy cost of flight, which is far higher than can be sustained for prolonged periods except for a few bird species with special adaptations to gliding flight such as albatrosses and frigatebirds [11] [12]. Empirical evidence from many sources shows that all birds can only increase sustainable energy expenditure up to a limit of four times Basal Metabolic Rate (BMR) [13]. The development of a metabolic approach to setting an upper limit to flight activity is explored here.

The energy expenditure of birds during breeding has been measured for a wide range of species by use of the doubly labelled water method [14]. The daily energy expenditure (kj/day) during breeding (known as Field Metabolic Rate, FMR) is expressed by the equation:

$$
\mathrm{FMR}=10.5 \mathrm{M}^{0.681}
$$

This has been tested for a large sample of bird species and with a high statistical confidence $\left(\mathrm{n}=95, \mathrm{r}^{2}=\right.$ 0.938, p < 0.0001) [14], so can be estimated for any bird species for which it has not been measured directly, from a knowledge of the mean weight of birds. Although birds can expend energy at higher rates, for example by using fat stores to fuel migratory flight, such rates of energy expenditure require metabolism of stored reserves and so are not sustainable.

The maximum sustainable work rate of birds (and of mammals) has been shown to be four times BMR, a ceiling that is set by physiological constraints on energy processing [13]-[17]. Flight represents a critical part of the daily energy budget of flying birds because flight costs are especially high and particularly so in birds that are larger than typical small passerines, so that the amount of time birds can spend in flight within their maximum sustainable work rate is constrained [12] [17]-[22]. 


\subsection{Setting the Flight Time Ceiling}

Where flight is by flapping flight at relatively high speed and without major use of economies of soaring, the mean energy cost of sustained flight is around 10 times BMR [12] [17]-[19] [21]-[24]. Numerous short flights, or flight with frequent changes of direction and altitude, as in display flights or territorial or courtship chases, will have higher energy costs [19] [23] [24]. Birds with high wing loading also have higher flight energy costs than birds with low wing loading [12] [18]. The mean cost of flapping flight for birds with high wing loading is therefore around 12 times BMR whereas for birds with low wing loading it is around 8 times BMR [12] [18] [24]. There is, therefore, a limit to the amount of time that a bird can spend in flight, especially display flights, and remain within the ceiling of four times BMR. Since most other routine activities such as foraging, walking, preening, resting, incubating eggs, digesting food, and general maintenance behaviour cost 2.6 times BMR [13] [20] [25], this sets a maximum of no more than three hours per day in flight in order to keep total daily (24 hours) metabolism below the ceiling of four times BMR for species with high wing loading [12] [18]. An example of this calculation for a high wing-loading species, assuming 3 hours of flapping flight (at 12 BMR) and 21 hours of other activity (at 2.6 BMR) is shown in Equation (2):

$$
24 \times 4 \mathrm{BMR} \approx(21 \times 2.6)+(3 \times 12)
$$

This theoretical conclusion was demonstrated empirically by experimental work with starlings Sturnus vulgaris, a species with relatively high wing loading. Breeding adult starlings declined in body mass as they were forced to spend more than two hours per day in flight, and this decline increased dramatically when birds had to spend three hours per day in flight, indicating that this work rate had exceeded a sustainable level [13]. This interpretation has been supported by subsequent studies, for example demonstrating that kestrels Falco tinnunculus forced experimentally to increase work rate above four hours flight per day incurred a large survival penalty [15].

For bird species with a low wing loading, and slow flapping flight, where the metabolic cost of flight is around 8 times BMR, the same consideration indicates a ceiling of no more than six hours of flight per day as shown in Equation (3):

$$
24 \times 4 \mathrm{BMR} \approx(18 \times 2.6)+(6 \times 8)
$$

While birds may be able to exceed the mean four times BMR ceiling for a short period, this will be at the cost of depleting body stores such as fat and protein, and so would be likely to compromise survival prospects. It is, therefore, reasonable to assume that birds will generally avoid exceeding the four times BMR ceiling except in the short term and for brief periods. Some species, such as pelagic seabirds, are adapted to cope with fluctuations in foraging conditions by carrying large fat reserves even when breeding [26], but this does not apply to most bird species. Although migratory birds may store large quantities of fat to fuel long-distance migrations [27], they do not carry large reserves of fat when breeding [28], and indeed the evidence is that most birds regulate body stores of energy at levels that reduce risk of starvation but minimize increase in body mass because higher body mass increases risk of capture by a predator [29].

Birds that use soaring or gliding flight will have a lower flight energy cost, so will be able to sustain higher periods of time in flight. In the extreme case of birds highly adapted to dynamic soaring, such as albatrosses, the energy cost of flight is below four times BMR [11], so that continuous flight is possible without reaching an energy-based constraint. The approach we propose would therefore not be applicable to such species because no ceiling to flight activity could be set. However, many bird species of conservation concern with regard to wind farm collision risk use flapping flight, and so there is an upper limit to the amount of time spent in flight, and therefore an assessment can be made of the collision risk associated with this flight activity.

The simplest form of ceiling set by energy costs would be to assume that birds work to their maximum capacity at all times. This would set a limit of three hours of flight per day by birds with a high wing loading that use flapping flight, or six hours of flight per day by birds with a low wing loading that use flapping flight. That approach would be precautionary since birds may not reach their metabolic ceiling all the time, and so if an assessment of collision risk based on such a model indicated no adverse effect on populations then there would be no need to carry out a more complex assessment based on knowledge of the natural history of the species. However, this metabolic ceiling approach can be combined with knowledge of the natural history of bird species in order to provide more realistic estimates, as described in the following sections. 


\subsection{Time Budgets Based on Natural History Knowledge}

The energy costs of birds are generally highest when rearing chicks [14], and for this reason, birds time their breeding so that chick-rearing coincides with the seasonal peak of food resources [30]. It is therefore during chick-rearing that birds are most likely to be working at sustained levels close to their physiological limits. Setting aside the seasonal flights of long-distance migrant species (for which specific stores of energy need to be accumulated), flight activity of birds is therefore likely to peak during chick-rearing. The amounts of flight activity can therefore be scaled to a peak during chick-rearing, taking account of natural history knowledge of the dates of arrival, laying, hatching, fledging and departure of birds, and seasonal changes in amounts of display flight and foraging activity during early and late breeding season relative to the peak of activity during chickrearing.

\section{A Case Study: Hen Harriers}

\subsection{The Requirement for Scenario Modelling}

It proved difficult to assess likely impacts of a proposed wind farm development at Stranoch adjacent to Glen App and Galloway Moors SPA in southern Scotland because the breeding population of hen harriers on this SPA was considerably depleted (in "unfavourable condition") and breeding success was low during the three years in which surveys of bird activity around the development site were carried out. Empirical evidence from Vantage Point surveys suggested little hen harrier activity in the proposed wind farm area, but provided no indication of how this might differ if the population was in "favourable condition" (defined as conditions similar to when the population was originally designated as an SPA).

Where an SPA population is below the levels for which it has been designated, the assessment needs to consider how a development may affect the population's ability to recover to its size at designation. SNH therefore advised that it would be necessary to demonstrate that the development will not be detrimental to the full recovery of the SPA, and that could not be achieved using empirical data on hen harrier flight activity at the site.

\subsection{Defining the Breeding Cycle of Breeding Hen Harriers}

Hen harriers start to visit breeding sites in southern Scotland during March, but pairing displays generally occur at the end of March, so establishment or re-establishment of nesting sitesoccurs in early April to late April, with egg laying starting from late April [31]. There is a 30 day incubation period which starts around laying of the third egg, followed by a 30 - 35 day nestling period, after which nestlings remain dependent for a further 2 - 4 weeks [31]. Thus a typical breeding attempt would involve 30 days of pre-laying activity centred around the nest site in April, followed by 31 days of egg laying and incubation during May, followed by 30 days of chick rearing in June, followed by 31 days of fledgling rearing in July when the young are still mostly fed at the nest site [32], with dispersal of the young in August.

\subsection{Defining the Foraging Range of Breeding Hen Harriers}

Radio tracking of breeding male hen harriers shows that their foraging activity is highest close to the nest site, and decreases with distance, with very few foraging trips taking birds more than $2 \mathrm{~km}$ from the nest [33] [34]. Radio tracking studies at Langholm, Orkney and Galloway [33] [34] estimated the ranging area used $90 \%$ of the time by male hen harriers as between 650 and 1180 ha (equivalent to foraging distances of $1.4 \mathrm{~km}$ and $1.9 \mathrm{~km}$, assuming circular foraging areas). Equivalent figures for females ranged up to a maximum of 350 ha (equivalent to a maximum foraging distance of about $1.1 \mathrm{~km}$ ).

Visual observations of breeding male hen harriers showed that they spend the majority of time foraging within $2 \mathrm{~km}$ of the nest site [33] [35] and only habitat within $2 \mathrm{~km}$ of the nest site influences the type of prey brought to the nest [36]. On the basis of the proportional effort reported from the tagging studies, the amount of time spent by foraging birds within bands $500 \mathrm{~m}$ wide radiating up to $2 \mathrm{~km}$ from the nest location was estimated.

\subsection{Defining the Flight Seconds of Breeding Male and Female Hen Harriers}

The main prey of breeding hen harriers is voles and small passerines such as meadow pipits Anthus pratensis. A vole weighing around $40 \mathrm{~g}$ provides about $200 \mathrm{~kJ}$, while a meadow pipit weighing around $19 \mathrm{~g}$ provides about 
$100 \mathrm{~kJ}$ (allowing for some indigestible content and the energy costs of assimilating food). Applying the Field Metabolic Rate Equation (1) to the hen harrier, a male weighs around $350 \mathrm{~g}$ [37], so has an estimated average field metabolic rate of $567 \mathrm{~kJ} / \mathrm{day}$. So a male hen harrier would need to eat about 3 voles per day, or about 6 meadow pipits a day to obtain its energy requirements. Brown [38] used empirical data on food consumption by hen harriers to conclude that their daily food requirement can be provided by about "two large voles or four small ones”. So Brown's estimate based on observed consumption of food by hen harriers agrees closely.

A nonbreeding hen harrier can easily obtain its daily food requirements in about an hour of hunting [38]. The energy budget of a breeding male hen harrier is greater as it includes food required for self-maintenance plus food provided to the female pre-laying in April to assist in egg formation, provided to the incubating female in May, and provided to the young in June and July. Peak flight activity of breeding males is therefore likely to occur in June-July.

Because hen harriers have a relatively low wing loading and fly slowly when hunting, their flight may be less energetically expensive than that of some birds which fly faster and have higher wing loadings. So a ceiling of between four and six hours of flight activity per day may be consistent with keeping the daily metabolic rate below four times BMR. This theoretical estimate is consistent with a small data set for tracked male hen harriers breeding in Ireland indicating that chick-rearing males flew for about four to six hours per day [39].

Females mainly guard the nest site, but spend smaller amounts of time foraging close to the nest. Males also spend small amounts of time in display flights early in the season, although these mostly involve birds that are establishing territories rather than established males.

Estimation of the amount of foraging activity (flight seconds) was therefore based on the following: Male foraging in April is one hour of flight activity per day, centred at the nest site and decreasing with distance from the nest; Male foraging in May is two hours of flight activity per day, centred at the nest site and decreasing with distance from the nest; Male foraging in June and July is six hours of flight activity per day, centred at the nest site and decreasing with distance from the nest.

\subsection{Using the Flight-Seconds Estimate to Generate a Theoretical Collision Risk Estimate}

Flight-seconds estimates were used in conjunction with a 17-year data set on breeding hen harriers within the Glen App and Galloway Moors, published data on hen harrier flight heights [40], and collision risk guidance from the regulator to generate a scenario based collision risk estimate. Using the data on breeding hen harriers for Glen App and Galloway Moors SPA it was possible to determine the frequency of nesting attempts within 2 $\mathrm{km}$ of the turbine closest to the SPA and an average nest location (average of eastings and northings) from which to estimate flight activity in the scenario model.

When considering how activity levels may relate to collision risk, about $90 \%$ of hen harrier flight activity occurs below potential collision risk height; the vast majority of flight activity taking place close to the ground [40]. Combining the estimated amount of time spent by hen harriers flying at rotor height in each $500 \mathrm{~m}$ band around the nest location with the proportion of each band which was swept by wind turbine rotors enabled estimation of the amount of time birds would be considered at risk of collision. Using the average flight speed this was then converted into an estimate of the number of passes made through the rotor swept area and multiplied by the probability of collision on any given transit (from the Band model [41]) to give an estimate of the number of collisions. These data permitted a collision risk analysis to be carried out for the hypothetical scenario where the hen harrier population had recovered to achieve favourable conservation status.

Empirical data collected from vantage point watches suggested 0.015 collisions per year but based on a depleted population of hen harriers during the survey period and breeding failure. By comparison, the energeticsbased modelling suggested 0.27 collisions per year for a population of the size present at SPA designation and with successful breeding. This estimate was below levels that would affect the integrity of the SPA and so allowed SNH to remove their holding objection to the development.

\section{A Case Study: Greenshanks}

\subsection{The Requirement for Scenario Modelling}

Vantage point surveys at a proposed wind farm site at Strathy South in Caithness, surrounded by the most important SPA for breeding greenshanks in the UK, recorded few flights by greenshanks despite the fact that large 
numbers were known to be breeding in the area. This was probably due in part to the cryptic nature of breeding greenshanks [42] [43].

Several aspects of greenshank biology make their flight activity budgets particularly difficult to observe. Greenshanks often nest several kilometres away from their feeding territory. They also defend a display area, which can be some distance away from their nest site and their preferred feeding area [43]. This behaviour appears to be an adaptation to minimize risk of nest predation, which is consistent with the cryptic activity of the species when breeding. Change-overs at the nest are very infrequent, with a tendency for females to incubate from very early morning to late evening, and males to incubate during the night [43]. As a result, flights between feeding areas and nests may occur predominantly very early in the morning and late at night [44]. During most of the day, when many vantage point surveys are carried out, there may be very little flight activity taking place. After hatching, adults may escort chicks long distances away from the nest site to suitable feeding areas, so that activity may occur in very different areas at different stages in the breeding season [43].

\subsection{Defining the Activity Budget of Greenshanks}

Although greenshank breeding behaviour has been studied in great detail [43] [44], there are no quantitative data on flight activity budgets, so the detailed qualitative information needed to be converted into quantitative scenarios to estimate collision risk. Greenshanks use direct and fast flapping flight, and have a high wing loading, so the energy cost of flight is likely to be high [12] [18]. Assuming that most other routine activities such as foraging, walking, preening, resting, incubating eggs, digesting food, and general maintenance behaviour costs about 2.6 times BMR [13] [20] [25] [45], this is likely to set a maximum of no more than three hours per day in flight for a bird the size of the greenshank in order to keep total metabolism below the ceiling of four times BMR [13].

This constraint of a maximum of three hours of flight per day allows construction of alternative activity budget scenarios based on the maximum sustainable amount of flight activity, a more realistic scenario with a lower level of flight activity, and a likely minimum scenario with a level of flight activity set at the minimum plausible. Comparison among scenarios then allows assessment of likely collision risks and the range of variation due to uncertainty in the approach.

The timing of the greenshank breeding season is well documented. Males generally arrive back on the breeding grounds in Scotland in late March or early April, with females following soon after [43] [46]. Males and females take part in display flights, though females less than males. Display flights may occur for several hours per day and may involve long chases over several km; birds breeding in treeless areas and at high population density seem to spend more time in display flight [44]. Most display activity occurs between 0300 and 0700 hrs [47]. Breeding birds usually fly at least $500 \mathrm{~m}$ from nest to feeding site, but often fly five $\mathrm{km}$, and may travel up to $14 \mathrm{~km}$ [44]. Display activity is much reduced in May while birds are incubating [44], although birds that lose the clutch may resume displaying and lay a replacement clutch.

Females normally lay four eggs, with intervals of about 44 hours between eggs [43]. Clutch completion varied from year to year and among studies but was mainly around 5th to 10th May [43]-[46], indicating that egg laying starts around 1st May. Incubation continues for 24 - 26 days [43] [44], and may be by both sexes although in many pairs most incubation is by the female. During incubation, which is predominantly by females during the day, females will make two (sometimes one to four) feeding trips per day of ca. 30 minutes, flying typically 2 $\mathrm{km}$ to feeding grounds [44]. Males will explore different possible feeding sites and will song-dance overhead to establish territorial ownership of suitable feeding areas [43]. Broods may move several kilometres from nest to feeding grounds, depending on local geography. Chicks fledge when about 29 days old [44], so start to fly around the beginning of July. Females tend to migrate south first (early to mid-July). Shortly after chicks can fly strongly, males and fledglings migrate south (mid to end of July or early August) [43] [46].

\subsection{Defining Flight-Seconds of Breeding Greenshanks}

Flight activity can therefore be estimated in relation to the following phenology: males present for 30 days in April (display and foraging), 31 in May (incubation period), 30 in June (chick-rearing) and 20 in July (tending fledglings). Females present for 30 days in April (display and foraging), 31 in May (incubation period), 30 in June (chick-rearing) and 10 in July (tending fledglings). Fledglings present for 20 days in July.

Females spend less time in display flight activity than males, but probably spend more time in commuting flights than males as they need to feed more intensely to obtain resources for egg formation and are likely, on 
average, to do this in a larger number of foraging bouts than required by males. In May, incubation requires females to commute between the nest and feeding areas, and the constraint of having to return to the nest site after each foraging bout will increase their commuting costs relative to the pre-laying period. The need to visit the nest site as well as feeding areas and to display will probably increase commuting flight activity of males in May compared to pre-laying levels in April, but display activity is known to decrease from peak pre-breeding levels so is assumed to be much less in May than in April.

When eggs hatch, commuting flight costs for males and females will reduce because chicks are led towards feeding grounds within the first few days after hatching. The modelling uses an estimated $50 \%$ reduction in commuting flight activity between incubation (May) and chick-rearing (June), but assumes a small increase in the amount of display/territorial defence flight activity because movement of chicks to feeding grounds results in adult territorial behaviour (i.e. display flights) over the area being used by chicks. In July, when chicks are larger, adult flight activity associated with commuting between sites is assumed to reduce further, whereas display/territorial activity is assumed to continue but at a lower level, at half the level in June.

Fledglings begin flying in July. The literature provides very little guidance as to how much time fledglings spend in flight in the breeding areas in July before dispersing away from the area in late July. For the modelling it is assumed that fledglings spend between 0 and 60 minutes in flight per day during July, but the most likely amount seems to be towards the lower end of this range. In 60 minutes of flight a fledgling flying at an average of $10 \mathrm{~m} / \mathrm{sec}$ could travel $36 \mathrm{~km}$. While it is known that fledglings will explore their natal area before dispersing, it seems very unlikely that fledglings would explore over such large distances on a daily basis.

Greenshank sustained level flight speed is around $12.3 \mathrm{~m} / \mathrm{sec}$ [48]. However, birds need to take off and land, and may need to gain height during flights between nest sites and feeding sites, so commuting flights of only $500 \mathrm{~m}$ to a few $\mathrm{km}$ will average a slower speed than this, perhaps around $10 \mathrm{~m} / \mathrm{sec}$ for a typical commuting flight. The activity budget assumes that, during April, males commute between display sites, foraging sites and potential nest sites, typically making three return trips of ca $3 \mathrm{~km}$ (1.5 km each way based on the typical distance between nest sites and foraging sites [43] [44]), at an average flight speed of $10 \mathrm{~m} / \mathrm{sec}$, so spend about $15 \mathrm{mi}-$ nutes flying. Around this estimate, are possible minimum estimates of only six minutes commuting, and maximum of 30 minutes commuting, based on descriptions of the minimum and maximum distances between nest sites and feeding sites [43] [44]. The large difference between the lower and upper estimates is due to high uncertainty in the evidence, and also to the indication in the literature that these values vary considerably among pairs and among locations, at least in part likely to be a result of local ecological features and the spatial scale of habitat mosaics.

Males show considerable song-flight activity in April and may chase other males and females in display flights. Such flights involve high aerial agility, but are likely to average around $10 \mathrm{~m} / \mathrm{sec}$. The amount of time spent in display flight is uncertain, but may be around 90 minutes per day. A minimum of 60 minutes per day is assumed. A ceiling on all flight activity is set by the metabolic cost which will limit this to no more than three hours total in flight per day for both commuting and display, so, allowing for up to 30 minutes of commuting, a maximum of about 150 minutes in display flight is assumed for days in April.

Females spend less time in display flight activity than males, but probably spend more time in commuting flights than males as they need to feed more intensely to obtain resources for egg formation and are likely, on average, to do this in a larger number of foraging bouts than required by males. In May, incubation requires females to commute between the nest and feeding areas, and the constraint of having to return to the nest site after each foraging bout will increase their commuting costs relative to the pre-laying period. The need to visit the nest site as well as feeding areas and to display will probably increase commuting flight activity of males in May compared to pre-laying levels in April, but display activity is known to decrease from peak pre-breeding levels so is assumed to be much less in May than in April. When eggs hatch, commuting flight costs for males and females will reduce because chicks are led towards feeding grounds within the first few days after hatching. The modelling uses an estimated 50\% reduction in commuting flight activity between incubation (May) and chickrearing (June), but assumes a small increase in the amount of display/territorial defence flight activity because movement of chicks to feeding grounds results in adult territorial behaviour (i.e. display flights) over the area being used by chicks. In July, when chicks are larger, adult flight activity associated with commuting between sites is assumed to reduce further, whereas display/territorial activity is assumed to continue but at a lower level, at half the level in June.

Fledglings begin flying in July. The literature provides very little guidance as to how much time fledglings 
spend in flight in the breeding areas in July before dispersing away from the area in late July. For the modelling it is assumed that fledglings spend between 0 and 60 minutes in flight per day during July, but the most likely amount seems to be towards the lower end of this range. In 60 minutes of flight a fledgling flying at an average of $10 \mathrm{~m} / \mathrm{sec}$ could travel $36 \mathrm{~km}$. While it is known that fledglings will explore their natal area before dispersing, it seems very unlikely that fledglings would explore over such large distances on a daily basis.

\subsection{Using the Flight-Seconds Estimate to Generate a Theoretical Collision Risk Estimate}

Estimation of the amount of flight activity was therefore based on three scenarios, summarised in Table 1. Those amounts then sum to minutes of flight activity, summarised in Table 2. From these data, standard methods can be used to assess collision risk for the three scenarios which provide a high, low and mid-range estimate

Table 1. Assumed flight activity budgets of greenshanks in terms of time spent commuting between nest sites and foraging areas, and in terms of time spent in territorial or sexual display flights, in minutes flight per day, of adult males, adult females, and fledglings, based on three scenarios; high level set by maximum sustainable metabolic level, low based on the minimum flight activity consistent with knowledge of breeding behaviour, and average set at a level intermediate between these extremes.

\begin{tabular}{|c|c|c|c|c|c|c|c|c|c|}
\hline \multirow{2}{*}{ Class } & \multirow{2}{*}{$\begin{array}{l}\text { Activity budget } \\
\text { levels }\end{array}$} & \multicolumn{4}{|c|}{$\begin{array}{l}\text { Mean minutes in flight per day } \\
\text { (commuting to forage) }\end{array}$} & \multicolumn{4}{|c|}{$\begin{array}{l}\text { Mean minutes in flight per day } \\
\text { (display) }\end{array}$} \\
\hline & & April & May & June & July & April & May & June & July \\
\hline \multirow{3}{*}{ Males } & Low & 6 & 12 & 6 & 3 & 60 & 10 & 30 & 15 \\
\hline & Average & 15 & 30 & 15 & 10 & 90 & 20 & 60 & 30 \\
\hline & High & 30 & 60 & 30 & 20 & 150 & 40 & 120 & 60 \\
\hline \multirow{3}{*}{ Females } & Low & 6 & 12 & 6 & 3 & 0 & 0 & 0 & 0 \\
\hline & Average & 30 & 45 & 20 & 10 & 10 & 10 & 10 & 5 \\
\hline & High & 60 & 90 & 45 & 20 & 30 & 30 & 30 & 15 \\
\hline \multirow{3}{*}{ Fledgling } & Low & 0 & 0 & 0 & 0 & 0 & 0 & 0 & 0 \\
\hline & Average & 0 & 0 & 0 & 10 & 0 & 0 & 0 & 0 \\
\hline & High & 0 & 0 & 0 & 60 & 0 & 0 & 0 & 0 \\
\hline
\end{tabular}

Table 2. Assumed flight activity budgets of greenshanks in terms of total minutes flight per day, of adult males, adult females, and fledglings (based on components in Table 1).

\begin{tabular}{|c|c|c|c|c|c|}
\hline \multirow{2}{*}{ Class } & \multirow{2}{*}{$\begin{array}{l}\text { Activity budget } \\
\text { levels }\end{array}$} & \multicolumn{4}{|c|}{$\begin{array}{l}\text { Mean minutes in flight per day } \\
\text { (commuting to forage) }\end{array}$} \\
\hline & & April & May & June & July \\
\hline \multirow{3}{*}{ Males } & Low & 66 & 22 & 36 & 18 \\
\hline & Average & 105 & 50 & 75 & 40 \\
\hline & High & 180 & 100 & 150 & 80 \\
\hline \multirow{3}{*}{ Females } & Low & 6 & 12 & 6 & 3 \\
\hline & Average & 40 & 55 & 30 & 15 \\
\hline & High & 90 & 120 & 75 & 35 \\
\hline \multirow{3}{*}{ Fledgling } & Low & 0 & 0 & 0 & 0 \\
\hline & Average & 0 & 0 & 0 & 10 \\
\hline & High & 0 & 0 & 0 & 60 \\
\hline
\end{tabular}


[49]. This modelling allowed SNH to take a clear position regarding the impact of the proposed Strathy South wind farm on the Caithness and Sutherland Peatlands SPA at the public enquiry, giving much greater confidence than had been provided by vantage point survey data.

\section{Discussion}

In the case studies outlined above, an energetics approach combined with knowledge of the natural history of the species has allowed theoretical scenarios to be tested to evaluate likely collision risk. This approach was helpful to the developers and statutory nature conservation advisors in presenting a range of plausible outcomes despite the short-comings of empirical vantage point data on flight activity budgets. This approach has enabled uncertainty within the assessment to be adequately addressed by using the best available scientific knowledge and in turn this has allowed informed decisions to be made by competent authorities.

We suggest that this approach may be broadly applicable to certain birds of particular conservation concern at many onshore and offshore wind farm developments, and therefore represents a useful tool contributing to HRA, EcIA and also potentially Strategic Environmental Assessments, SEA (2001/42/EC).

In particular, this approach could be of use in a number of situations including: where SPA populations are in unfavourable condition; where flight activity is difficult to observe due to behavioural characteristics; for modelling the impact of land-use change (forest felling/restructuring); where historic regularly used nest sites are not used during vantage point surveys; where breeding fails and vantage point surveys are therefore not representative of a season where breeding is successful; or as an early risk assessment tool by developers. There is also scope to use this type of scenario modelling in Strategic Environmental Assessments (SEAs). For example, SEAs for offshore wind consenting rounds could consider impacts on seabird SPA populations using scenario modelling to ensure the best available approaches are used to inform the appropriate assessment for the proposed Plan.

In the absence of knowledge about the natural history of the species, a precautionary approach would be to assume that birds work at their energetic ceiling all the time. This would define a maximum plausible level of flight activity, and therefore would indicate a maximum plausible level of collision mortality. However, more refined estimates can be derived where there is information on the natural history of the species, allowing more realistic assessment of seasonal variation in the activity budget of birds with the metabolic ceiling used to define the seasonal peak of flight activity.

The development of new techniques is allowing the time budgets of birds to be quantified by deployment of data loggers on a sample of individuals. This will make it increasingly possible to obtain species-specific empirical evidence of the amount of time spent in flight, and therefore at potential risk of collision with wind turbines. Such evidence will not only provide greater confidence in the use of an energetics constraint modelling approach to assessment of risk, but would also permit assessment of collision risk for bird species that use soaring flight (such as golden eagles Aquila chrysaetos). In such species, the amount of time birds can spend flying may not be so clearly constrained by energetic considerations as seen in species that use flapping flight, but could be measured empirically. Furthermore, it may be possible to refine models further through the addition of quantitative spatial and temporal data on prey availability and habitat suitability. We therefore strongly advocate this general approach as a useful new tool in the assessment of collision risk and population-level impacts of wind farms on birds, and as an approach that is complementary to traditional vantage point survey and collision assessments.

\section{Acknowledgements}

We thank SNH colleagues for expert advice and discussion on the approach developed in this paper, especially Andrew Stevenson and Paul Taylor for discussions on the hen harrier modelling, and Des Thompson for advice on greenshank ecology. Paul Haworth kindly made available a copy of his unpublished report on impacts of wind farms on hen harriers. Mark Wilson and John O’Halloran kindly provided unpublished information on their tracking studies of hen harriers in Ireland.

\section{References}

[1] European Commission (2011) Energy Roadmap 2050.COM885/2. 
[2] Federal Ministry of Economics and Technology (2010) Energy Concept for an Environmentally Sound, Reliable and Affordable Energy Supply. Federal Ministry of Economics and Technology, Berlin.

[3] European Wind Energy Association (2015) Wind in power 2014 European Statistics. European Wind Energy Association, Brussels.

[4] Drewitt, A.L. and Langston, R.H.W. (2006) Assessing the Impacts of Wind Farms on Birds. Ibis, 148, $29-42$. http://dx.doi.org/10.1111/j.1474-919X.2006.00516.x

[5] Dahl, E.L., Bevanger, K., Nygard, T., Roskaft, E. and Stokke, B.G. (2012) Reduced Breeding Success in White-Tailed Eagles at Smøla Windfarm, Western Norway, Is Caused by Mortality and Displacement. Biological Conservation, 135, 79-85. http://dx.doi.org/10.1016/j.biocon.2011.10.012

[6] Scottish Natural Heritage (2013) Recommended Bird Survey Methods to Inform Impact Assessment of Onshore Wind Farms. SNH Guidance. Scottish Natural Heritage, Battleby.

[7] Fielding, A., Haworth, P., Whitfield, P., McLeod, D. and Riley, H. (2011) A Conservation Framework for Hen Harriers in the United Kingdom. JNCC Report No. 441. Joint Nature Conservation Committee, Peterborough.

[8] Natural England (1992) EC Directive 79/409 on the Conservation of Wild Birds: Special Protection Area Bowland Fells (Lancashire). Secretary of State for the Environment, London.

[9] Hayhow, D.B., Eaton, M.A., Bladwell, S., Etheridge, B., Ewing, S.R., Ruddock, M., et al. (2013) The Status of the Hen Harrier, Circus Cyaneus, in the UK and Isle of Man in 2010. Bird Study, 60, 446-458. http://dx.doi.org/10.1080/00063657.2013.839621

[10] SNH Sitelink. https://gateway.snh.gov.uk/sitelink/

[11] Shaffer, S.A., Costa, D.P. and Weimerskirch, H. (2003) Foraging Effort in Relation to the Constraints of Reproduction in Free-Ranging Albatrosses. Functional Ecology, 17, 66-74. http://dx.doi.org/10.1046/j.1365-2435.2003.00705.x

[12] Pennycuick, C.J. (2008) Modelling the Flying Bird. Elsevier Academic Press, Amsterdam and London.

[13] Drent, R.H. and Daan, S. (1980) The Prudent Parent: Energetic Adjustments in Avian Breeding. Ardea, 68, $225-252$.

[14] Nagy, K.A. (2005) Field Metabolic Rate and Body Size. Journal of Experimental Biology, 208, 1621-1625. http://dx.doi.org/10.1242/jeb.01553

[15] Moore, S.J. (Ed.) (2005) Seeking Nature’s Limits: Ecologists in the Field. KNNV Publishing, Utrecht.

[16] Green, J.A., Aitken-Simpson, E.J., White, C.R., Bunce, A., Butler, P.J. and Frappell, P.B. (2013) An Increase in Minimum Metabolic Rate and Not Activity Explains Field Metabolic Rate Changes in a Breeding Seabird. Journal of Experimental Biology, 216, 1726-1735. http://dx.doi.org/10.1242/jeb.085092

[17] Johnston, D.W. and McFarlane, R.W. (1967) Migration and Bioenergetics of Flight in Pacific Golden Plover. Condor, 69, 156-168. http://dx.doi.org/10.2307/1366605

[18] Pennycuick, C.J. (1989) Bird Flight Performance—A Practical Calculation Manual. Oxford University Press, Oxford.

[19] Nudds, R.L. and Bryant, D.M. (2000) The Energetic Cost of Short Flights in Birds. Journal of Experimental Biology, 203, 1561-1572.

[20] Rogers, D.I., Piersma, T. and Hassell, C.J. (2006) Roost Availability May Constrain Shorebird Distribution: Exploring the Energetic Costs of Roosting and Disturbance around a Tropical Bay. Biological Conservation, 133, 225-235. http://dx.doi.org/10.1016/j.biocon.2006.06.007

[21] Schmidt-Wellenburg, C.A., Biebach, H., Daan, S. and Visser, G.H. (2007) Energy Expenditure and Wing Beat Frequency in Relation to Body Mass in Free Flying Barn Swallows (Hirundorustica). Journal of Comparative Physiology B, 177, 327-337. http://dx.doi.org/10.1007/s00360-006-0132-5

[22] Weber, J.M. (2009) The Physiology of Long-Distance Migration: Extending the Limits of Endurance Metabolism. Journal of Experimental Biology, 212, 593-597. http://dx.doi.org/10.1242/jeb.015024

[23] Butler, P.J. and Bishop, C.M. (2000) Flight. In: Whittow, G.C., Ed., Sturkie’s Avian Physiology, Chap. 15, Academic Press, London, 391-435. http://dx.doi.org/10.1016/B978-012747605-6/50016-X

[24] Gavrilov, V.M. (2011) Energy Expenditures for Flight, Aerodynamic Quality, and Colonization of Forest Habitats by Birds. The Biological Bulletin, 38, 779-788. http://dx.doi.org/10.1134/S1062359011080024

[25] Piersma, T., Dekinga, A., Van Gils, J.A., Achterkamp, B. and Visser, G.H. (2003) Cost-Benefit Analysis of Mollusc Eating in a Shorebird. 1. Foraging and Processing Costs Estimated by the Doubly Labelled Water Method. Journal of Experimental Biology, 206, 3361-3368. http://dx.doi.org/10.1242/jeb.00545

[26] Jacobs, S.R., Edwards, D.B., Ringrose, J., Elliott, K.H., Weber, J.M. and Gaston, A.J. (2011) Changes in Body Composition during Breeding: Reproductive Strategies of Three Species of Seabirds under Poor Environmental Conditions. Comparative Biochemistry and Physiology Part B, 158, 77-82. http://dx.doi.org/10.1016/j.cbpb.2010.09.011

[27] Newton, I. (2010) Bird Migration. HarperCollins, London. 
[28] Furness, R.W. and Greenwood, J.J.D. (1993) Birds as Monitors of Environmental Change. Chapman \& Hall, London. http://dx.doi.org/10.1007/978-94-015-1322-7

[29] Metcalfe, N.B. and Ure, S.E. (1995) Diurnal Variation in Flight Performance and Hence Potential Predation Risk in Small Birds. Proceedings of the Royal Society of London B, 261, 395-400. http://dx.doi.org/10.1098/rspb.1995.0165

[30] Gienapp, P., Lof, M., Reed, T.E., McNamara, J., Verhulst, S. and Visser, M.E. (2013) Predicting Demographically Sustainable Rates of Adaptation: Can Great Tit Breeding Time Keep Pace with Climate Change? Philosophical Transactions of the Royal Society B, 368, 1-10.

[31] Forrester, R.W., Andrews, I.J., McInerny, C.J., Murray, R.D., McGowan, R.Y., Zonfrillo, B., et al. (2007) The Birds of Scotland. Scottish Ornithologists Club, Aberlady.

[32] Newton, I. (1979) Population Ecology of Raptors. T \& AD Poyser, Berkhamsted.

[33] Arroyo, B., Leckie, F., Amar, A., Aspinall, D., McCluskie, A. and Redpath, S. (2004) Habitat Use and Range Management on Priority Areas for Hen Harriers: 2003 Report. CEH Project Number: C02018, NERC/Centre for Ecology \& Hydrology, 31 p.

[34] Arroyo, B.E., Leckie, F. and Redpath, S.M. (2006) Habitat Use and Range Management on Priority Areas for Hen Harriers: Final Report. CEH Report to SNH.

[35] Arroyo, B., Amar, A., Leckie, F., Buchanan, G.M., Wilson, J.D. and Redpath, S. (2009) Hunting Habitat Selection by Hen Harriers on Moorland: Implications for Conservation Management. Biological Conservation, 142, 586-596. http://dx.doi.org/10.1016/j.biocon.2008.11.013

[36] Amar, A., Arroyo, B.E., Redpath, S.M. and Thirgood, S. (2004) Habitat Predicts Losses of Red Grouse to Individual Hen Harriers. Journal of Applied Ecology, 41, 305-314. http://dx.doi.org/10.1111/j.0021-8901.2004.00890.x

[37] BTO BirdFacts. http://www.bto.org/about-birds/birdfacts

[38] Brown, L. (1976) British Birds of Prey. Collins, London.

[39] Professor John O’Halloran, Personal Communication.

[40] Haworth, P. and Fielding, A. (2014) A Review of the Impacts of Terrestrial Wind Farms on Breeding and Wintering Hen Harriers. Draft Report to Scottish Natural Heritage. Haworth Conservation, 34 p.

[41] Band, W., Madders, M. and Whitfield, D.P. (2007) Developing Field and Analytical Methods to Assess Avian Collision Risk at Wind Farms. In: De Lucas, M., Janss, G. and Ferrer, M., Eds., Birds and Wind Power, Quercus Editions, Madrid, 259-275. www.quercus.pt

[42] Hancock, M.H., Gibbons, D.W., Thompson, P.S. (1997) The Status of Breeding Greenshank (Tringanebularia) in the United Kingdom in 1995. Bird Study, 44, 290-302. http://dx.doi.org/10.1080/00063659709461064

[43] Nethersole-Thompson, D. and Nethersole-Thompson, M. (1979) Greenshanks. T \& AD Poyser, Berkhamsted.

[44] Nethersole-Thompson, D. (1951) The Greenshank. Collins New Naturalist, London.

[45] Hale, W.G. (1980) Waders. Collins New Naturalist, London.

[46] Thompson, D.B.A., Thompson, P.S. and Nethersole-Thompson, D. (1986) Timing of Breeding and Breeding Performance in a Population of Greenshanks (Tringanebularia). Journal of Animal Ecology, 55, 181-199. http://dx.doi.org/10.2307/4701

[47] Pendlebury, C., Zisman, S., Walls, R., Sweeney, J., McLoughlin, E., Robinson, C., et al. (2011) Literature Review to Assess Bird Species Connectivity to Special Protection Areas. Scottish Natural Heritage Commissioned Report No. 390.

[48] Alerstam, T., Rosen, M., Bäckman, J., Ericson, P.G.P. and Hellgren, O. (2007) Flight Speeds among Bird Species: Allometric and Phylogenetic Effects. PLoS Biology, 5, e197. http://dx.doi.org/10.1371/journal.pbio.0050197

[49] MacArthur Green (2016) Greenshank Collision Mortality Estimated Based on Ecological and Behavioural Studies. SNH Commissioned Research Report. (in press)

http://www.snh.gov.uk/publications-data-and-research/publications/search-the-catalogue/ 